\title{
The Effect of Dominant Ventricular Morphology on Outcomes of Single Ventricular Abnormalities: A Retrospective Analysis of Right Ventricle Versus Left Ventricle
}

\section{(1) Çağatay Bilen, (1) Gökmen Akkaya, (® Osman Nuri Tuncer, (1) Yüksel Atay}

Ege University Faculty of Medicine, Department of Cardiovascular Surgery, İzmir, Turkey

\begin{abstract}
Objectives: Within this study, we aimed to evaluate our single institute experience in patients to whom the Fontan procedure was performed; furthermore, to assess the effects of ventricle dominancy on peri-operative and postoperative findings, comorbidities, and survival.

Materials and Methods: Patient data were obtained from cardiac surgery database, anesthesia records, and hospital medical records. Patients were divided into two groups according to the ventricle dominancy following transthoracic echocardiography or cardiac catheterization findings. The first group had left ventricle (LV) with or without rudimentary right ventricle (RV) while the second group had rudimentary LV with or without RV.
\end{abstract}

Results: Chylothorax was observed in four patients in the RV group and in two patients in the LV group as well. However, this difference was not significant $(\mathrm{p}=0.296)$. On the other hand, when two groups were compared in terms of the length of pleural effusion, the RV group demonstrated statistically higher duration $(\mathrm{p}=0.028)$.

Conclusion: We did not observe any statistically significant adverse effect in patients undergoing the Fontan procedure according to ventricle dominancy except for prolonged pleural effusion. As a consequence, we state that ventricle dominancy does not affect early postoperative outcomes.

Keywords: Fontan procedure, dominant ventricle, congenital heart surgery

Address for Correspondence: Çağatay Bilen, Ege University Faculty of Medicine, Department of Cardiovascular Surgery, İzmir, Turkey Phone: +90 5556253603 e-mail: cagataybilen86@gmail.com ORCID: orcid.org/0000-0002-9158-5627

Received: 06.08.2019 Accepted: 31.10.2019

Cite this article as: Bilen Ç, Akkaya G, Tuncer ON, Atay Y. The Effect of Dominant Ventricular Morphology on Outcomes of Single Ventricular Abnormalities: A Retrospective Analysis of Right Ventricle Versus Left Ventricle. EJCM 2019;7(4):172-178. DOI: 10.32596/ejcm.galenos.2019.08.044 


\section{Introduction}

The Fontan circulation provides a direct venous flow from the caval veins towards the pulmonary arteries and since it was initially described by Fontan and Baudet ${ }^{(1)}$ in 1971, various modifications have been developed, and therefore, mortality rates substantially improved ${ }^{(2-3)}$. Although underlying etiology considerably varies among these patients, particularly Fontan surgery has a feature of being the last step for patients who have a functional single ventricle. However, such patients can be classified under two main groups according to which ventricle is dominant. Prior studies have revealed worse early and late-term outcomes including increased hospital death, interstage mortality, reduced functional capacity, and survival rate following Fontan surgery in the right dominant ventricle group ${ }^{(4,5)}$. These results have been interpreted as right ventricular structures are less equipped to maintain systemic circulation and thus, cause univentricle failure development in late term ${ }^{(6,7)}$. Although systemic right ventricle (RV) and tricuspid valve have been demonstrated to be inferior in other congenital heart diseases, for instance, the patients had undergone atrial switch with the diagnosis of dextro-transposition of the great arteries or physiologic repair performed ones with corrected transposition of the great arteries ${ }^{(8)}$. Nevertheless, there are limited studies investigating the influence of ventricle dominancy in this issue. Moreover, some authors indicate that recent progress in intensive care unit (ICU), developed technologies, and improved patient management lead to enhance outcomes in all stages, especially in hypoplastic left heart syndrome; therefore, such results might be associated with underlying cardiac morphology rather than the ventricle dominancy ${ }^{(9)}$. Within this study, we aimed to evaluate our single institute experience in patients to whom the Fontan procedure was performed; furthermore, to assess the effects of ventricle dominancy on perioperative and postoperative findings, comorbidities, and survival.

\section{Materials and Methods}

Patient data were obtained from cardiac surgery database, anesthesia records, and hospital medical records.
Nonetheless, pre-operative data were obtained from patient characteristics, echocardiography results, and cardiac catheterization reports. The operation data included blood transfusion requirement, inotrope requirement at cardiopulmonary bypass (CPB) termination, and durations of CPB and aortic cross clamp. Therewithal, extubation timing, lengths of hospital and ICU stay, duration of pleural effusion, presence/absence of Chylothorax, and rates of morbidity and mortality were evaluated in postoperative period. Peri-operative major adverse events were determined as cardiac arrest, neurological disorders, acute renal failure requiring hemodialysis or peritoneal dialysis, arrhythmias requiring permanent pacemaker, and multiple organ failure.

Patients were divided into two groups according to the ventricle dominancy following transthoracic echocardiography (TTE) or cardiac catheterization findings. The first group had left ventricle (LV) with or without rudimentary right ventricle while the second group had rudimentary LV with or without right ventricle.

Hematocrit value was attempted to be kept above $25 \%$. Nevertheless, in case of lactate increase, low urinary output or when the targeted mean arterial pressure could not be achieved despite adequate CPB flow which had been tempered to patient's weight and body temperature, allogenic blood transfusion was done. After weaning from $\mathrm{CPB}$, heparin was neutralized by protamine and then neutralization was controlled via active clothing time. In case of non-surgical hemorrhage was observed, the apheresis platelet suspension $(10 \mathrm{~mL} / \mathrm{kg})$ and cryoprecipitate $(1 \mathrm{~mL} / 10 \mathrm{~kg})$ were administered.

Hemogram, lactate concentration, electrolytes, ionized calcium, and coagulation profile values were measured after the patients were transferred from operation room to the ICU. An erythrocyte suspension was infused if the patient was observed as hypotensive or demonstrated increased metabolic acidosis and lactate concentration. If required, apheresis platelet suspension $(10 \mathrm{~mL} / \mathrm{kg})$ and cryoprecipitate $(1 \mathrm{~mL} / 10 \mathrm{~kg})$ were transfused to patients. 
When the amount of drainage was above $5 \mathrm{~mL} / \mathrm{kg} / \mathrm{h}$, blood transfusion was administered according to hemogram value and coagulation profile.

After discharge, the patients were kept in routine follow-up program by the surgical team for three months and the patients were examined via TTE in first and third months. Then, the follow-up period was continued by pediatric cardiologists.

All demographic and perioperative data were compared between two groups. Continuous data were presented with mean and standard deviation values. The comparisons between the two groups were performed using the MannWhitney $\mathrm{U}$ test for continuous data, and the chi-square test for categorical data. A p value of $<0.05$ was accepted as significant.

This study was approved by the local ethical committee with decision number $18-10.2 \mathrm{~T} / 43$ on $31 / 10 / 2018$. The informed consent form was obtained from each patient.

\section{Surgical Procedure}

Intra-extra cardiac Fontan modification was performed via cross clamp application under CPB. After mediastinal access was achieved through midline sternotomy, a Polytetrafluoroethylene (ePTFE) graft with appropriate size was anastomosed to the vena cava inferior orifice through right atriotomy incision. During procedure, a fenestration was created by using a $4 \mathrm{~mm}$ sized punch in a way to canalize the flow towards atrioventricular valve. Afterwards, right atrium was sewn to encircle the graft. Thereafter, bidirectional cava-pulmonary anastomosis was performed as usual.

Extra-cardiac Fontan procedure was performed in beating heart with $\mathrm{CPB}$ support. Vena cava inferior (VCI) was divided from the right atrium and then, right atrium was closed via running sutures. After ePTFE graft with appropriate size was anastomosed to VCI, standard bidirectional cava-pulmonary anastomosis was performed.

Regardless of which Fontan modification was used, same protocols were utilized in the operative room and ICU to the patients.
All patients operated between years of 2009 and 2014 underwent extracardiac Fontan modification. Afterwards, intra-extracardiac Fontan modification was performed to all patients; thereby, caused changes in our clinical approach.

Hydrochlorothiazide and spironolactone combination were administered in patients with diuretic treatment indication. None of the patients received angiotensinconverting enzyme inhibitors in the early period.

\section{Prolonged Pleural Effusion}

Chest tubes were removed if daily drainage was below $5 \mathrm{~mL} / \mathrm{kg}$. Prolonged pleural effusion was described as the effusions lasting more than 14 days or requiring re-intervention after removal of the initial chest tube.

\section{Chylothorax}

The presence of chylomicrons in pleural fluid or the detection of lymphocyte fraction of $80 \%$ or more in pleural liquid is being identified as Chylothorax. Pleural fluid samples were obtained on the postoperative fifth day in patients with chest tube drainage above $5 \mathrm{~mL} / \mathrm{kg} /$ day and then, chylomicron, lymphocyte fraction and triglyceride values were measured.

Patients with Chylothorax were fed orally with a medium-chain triglyceride form. Notwithstanding, in patients with ongoing Chylothorax, oral intake was stopped and was proceeded to total parenteral nutrition (TPN). Patients without improvement despite TPN were treated with $1 \mathrm{mg} / \mathrm{kg}$ prednisolone daily. Finally, octreotide (0.5-4 $\mathrm{mg} / \mathrm{kg} / \mathrm{hr}$ IV infusion) was added to the treatment protocol in patients with still ongoing Chylothorax. Patients who were receiving Chylothorax treatment were examined in terms of venous thrombosis formation by Doppler ultrasonography.

The treatment was terminated when the daily drainage was decreased below $2 \mathrm{~mL} / \mathrm{kg}$ and the chest tubes were removed, then patients were fed with cholesterol-poor diet for six weeks. 


\section{Results}

A total of 36 patients who underwent the Fontan procedure with CPB between 2009 and 2018 were included in the study. Aortic cross-clamp application was required in nine $(25 \%)$ patients who were treated with intra-extracardiac Fontan procedure. Of the patients, 11 were initially treated with modified Blalock-Taussig shunt, while 15 with pulmonary artery banding, and two with Norwood procedure as a palliative operation before Fontan operation. Patient characteristics were presented in Table 1.

The patients were divided into two groups as the LV dominant and right ventricle dominant groups. The groups were including 19 and 16 patients, respectively. The mean age was calculated as $12.97 \pm 20.66$ years and the mean weight was $18.77 \pm 7.64 \mathrm{~kg}$. Fenestration was performed in 18 patients. Epinephrine and/or milrinone support had to be administered in eight patients at the time of weaning from CPB. The mean CPB time was $81.44 \pm 27.81$ minutes.

Allogenic blood product was transfused in 10 patients during operation and four patients in ICU. All perioperative data regarding groups were shown in Table 2. All patients were evaluated by transesophageal echocardiography after weaning from $\mathrm{CPB}$. None of the patients had moderate or severe valve failure. Mortality was observed in one patient after the postoperative day 10 in the LV group and two in the RV group at postoperative day 6 and 12, respectively. Beyond that, there was no late mortality in regular follow-up period during the first three months following discharge. Major operative complications were concluded as arrhythmias requiring permanent pacemaker implantation, acute renal failure, and cerebrovascular events. While one patient in the RV group required a permanent pacemaker implantation due to severe arrhythmia, neurological deficit occurred in one patient in the LV group. Therewithal, none of those patients had acute renal failure.

The mean duration of intubation, intensive care, and hospital stay were $17.16 \pm 25$ hours, $59,2.41 \pm 0.96$ days and $11.19 \pm 4.08$ days, respectively. Chylothorax was observed in four patients in the RV group and in two patients in the LV group as well. However, this difference was not significant $(p=0.296)$. On the other hand, when two groups were compared in terms of the length of pleural effusion,

Table 1. Demographic findings

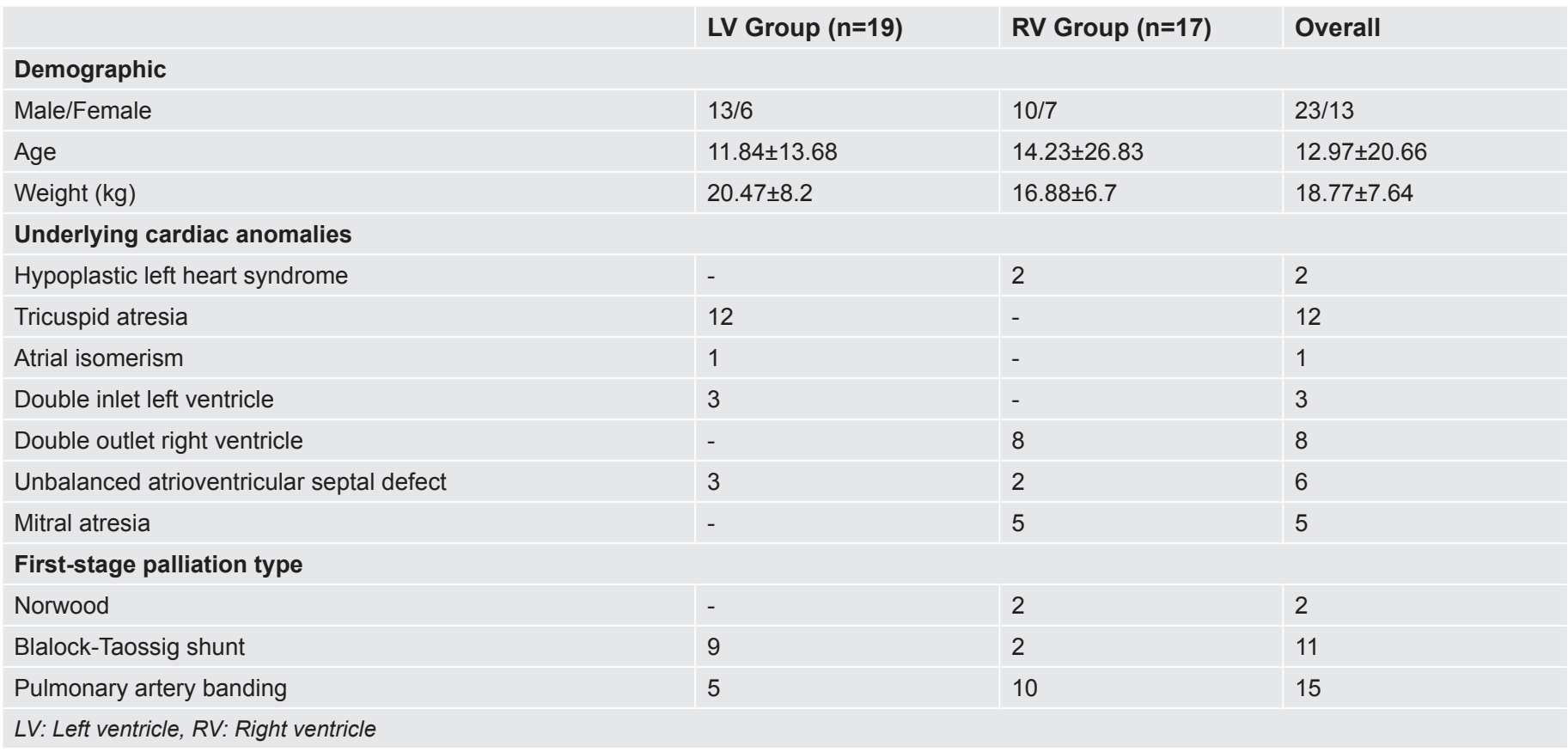


the RV group demonstrated statistically higher duration $(\mathrm{p}=0.028)$.

\section{Discussion}

The impact of ventricle dominancy on postoperative outcomes is still controversial in patients with a functionally univentricular heart undergoing a Fontan procedure hence it has been investigating for over decades. Although previous studies imply worse outcomes regarding the patients with dominant right ventricle, some recent studies have found no differences in the immediate

Table 2. Pre-operative and postoperative findings

\section{Pre-operative data}

Pre-operative hematocrit \%

Pre-operative oxygen saturation \%

Pre-operative creatinine $\mathrm{mg} / \mathrm{dL}$

Pre-operative mean pulmonary artery pressure

Intra-operative data

Use of cross-clamp in CPB $n(\%)$

Cross-clamping time ( $\mathrm{min}$ )

Cardiopulmonary bypass time (min)

Fenestration $\mathrm{n}(\%)$

Lowest hematocrit during surgery (\%)

Inotropic support at termination of CBP n (\%)

Intra-operative transfusion $\mathrm{n}(\%)$

\section{Postoperative data}

Postop transfusion n (\%)

Hematocrit level in admission to ICU

Lactate in admission to ICU

Pleural effusion time (day)

Chilothorax n (\%)

Duration of intubation (hour)

ICU length stay (day)

Hospital length stay (day)

Complications

Neurologic deficit, $n$ (\%)

Arrhythmia (permanent pacemaker needed, n (\%)

Sepsis, $n(\%)$

AKI within 7 days, $n(\%)$

Mortality intra-operative and postoperative course of these patients based on ventricular morphology ${ }^{(3-5,7,8)}$. Similarly, our current study supports latter findings by detecting no difference between the groups except for the length of pleural effusion.

In particular, hypoplastic left heart syndrome (HLHS) was held primarily responsible for the Fontan failure; furthermore, such patients had demonstrated reduced exercise capacity after Fontan surgery ${ }^{(3,5)}$. On the other hand, two studies conducted with larger series contradict these results. The University of Michigan ${ }^{(10)}$

\begin{tabular}{l|l|l} 
LV group (n=19) & RV group (n=17) & \multicolumn{1}{l}{$\mathbf{p}$} \\
\hline $48.47 \pm 1.67$ & $49 \pm 1.27$ & 0.436 \\
\hline $85.78 \pm 2.20$ & $85.32 \pm 2.49$ & 0.632 \\
\hline $0.35 \pm 0.03$ & $0.35 \pm 0.1$ & 0.675 \\
\hline $12.31 \pm 2.31$ & $13.7 \pm 3.54$ & 0.235 \\
\hline & & \\
\hline $4 / 19(21 \%)$ & $5 / 17(29 \%)$ & 0.563 \\
\hline $73 \pm 20.03$ & $70.6 \pm 15.85$ & - \\
\hline $72.73 \pm 25.11$ & $91.17 \pm 28.13$ & 0.063 \\
\hline $7 / 19(36.8 \%)$ & $11 / 17(64 \%)$ & 0.095 \\
\hline $26.42 \pm 1.21$ & $25.94 \pm 1.34$ & 0.27 \\
\hline $3 / 19(15.7 \%)$ & $5 / 17(29 \%)$ & 0.569 \\
\hline $4 / 19(21 \%)$ & $6 / 17(35 \%)$ & 0.341 \\
\hline & & \\
\hline $2 / 19$ & $2 / 17(11 \%)$ & 0.906 \\
\hline $39.36 \pm 1.42$ & $39.94 \pm 1.39$ & 0.278 \\
\hline $2.19 \pm 0.44$ & $2.25 \pm 0.51$ & 0.75 \\
\hline $4.58 \pm 4.77$ & $8.36 \pm 6.81$ & 0.028 \\
\hline $2 / 19(10.5 \%)$ & $4 / 17(23 \%)$ & 0.296 \\
\hline $18.1 \pm 25.80$ & $16.47 \pm 26.07$ & 0.080 \\
\hline $2.52 \pm 1.17$ & $2.29 \pm 0.68$ & 0.740 \\
\hline $10.23 \pm 5.73$ & $15.94 \pm 8.99$ & 0.074 \\
\hline $1(5 \%)$ & & \\
\hline $0(0 \%)$ & $0(0 \%)$ & 0.337 \\
\hline $1(5 \%)$ & $1(5 \%)$ & 0.284 \\
\hline $0(0 \%)$ & $1(5 \%)$ & 0.935 \\
\hline $1(5 \%)$ & $1(5 \%)$ & 0.284 \\
\hline & $2(11 \%)$ & 0.481 \\
\hline
\end{tabular}

LV: Left ventricle, RV: Right ventricle, CBP: Cardiopulmonary bypass, ICU: Intensive care unit, AKI: Acute kidney injury 
concluded after 15 years of follow-up experience in 636 patients that the Fontan procedure could be reliably performed regardless of which ventricle was dominant. Compatible with this, a group from Milwakuee have also reported their outcomes of 256 patients and emphasized that "ventricular morphology did not predict outcome" (11). However, Backer et al. declared that despite the fact that aforementioned studies revealedno statistical differences in the mean event-free survival at 10 years, considering these values were $75 \pm 7 \%$ in left and $67 \pm 8 \%$ in right ventricular group respectively ${ }^{(7)}$, these results may be interpreted in favor of the conclusion of d'Udekem et al. about that right ventricular dominance is a predictor of earlier mortality ${ }^{(3)}$. We observed no mortality in HLHS patients; thus, we agree with these authors although our study had comprised of limited sample size.

Another key aspect for these patients is the presence of increased adverse effect proportion. Iyengar et al. highlighted that despite excellent survival rate, patients with HLHS have higher risk of late adverse events than other morphological groups ${ }^{(12)}$. Furthermore, they detected a strong association with prolonged effusions. Although this study supports our findings, prolonged effusion was defined differently from ours as to describe the effusions longer than 30 days vs 14 days. They explained the reason why they deliberatively defined the prolonged effusion beyond the most accepted threshold was to isolate the truly troublesome effusions. Nonetheless, the utility of fenestration did not display a protective effect from pleural effusion formation even though a previous randomized study advocated the opposite ${ }^{(13)}$. We also did not observe better results with the application of fenestration.

In the same manner, McGuirk et al. have also found prolonged pleural effusion and increased duration of hospital stay in their serial including 103 (44 LV, 59 RV) patients ${ }^{(14)}$. Moreover, early survival and being free from re-operation or reintervention did not differ between the groups. Therewithal, they suggest that ventricular morphology may yet influence long term survival.

Besides that, Blinder et al. did not observe any perioperative complications including pleural effusion similar to Taylor et al. ${ }^{(15,16)}$. In a further analysis, the authors revealed close findings regarding 10 years of uneventful survival rates, and these were $75.2 \%$ in the HLHS group meanwhile $77.6 \%$ in the non-HLHS one.

One of the most important factors that lead to success in perioperative management is to avoid inadequate postoperative renal function as priorly remarked by Kamata et al. ${ }^{(8)}$. Several studies determined the relation between acute kidney injury (AKI) and prolonged need for mechanical ventilation and inotrope support, which thus caused prolonged stay in both the ICU and the hospital. We observed one AKI among our patients and that case had mortality after a prolonged duration of $\mathrm{ICU}^{(15,16)}$.

\section{Limitations of the Study}

This study includes inherent limitations due to its retrospective, non-randomized design. The study cohort was a heterogeneous population of patients undergoing a multistage surgical palliation process. Furthermore, this study period involves a 10-year period, over time the patient population have changed, and surgical practices have developed. For instance, we generally prefer fourth generation (intra-extracardiac) Fontan procedure and operating on more patients with dominant right ventricle. Beyond that, our results manifest early outcomes; therefore, further studies should be conducted in larger cohorts within prospective design in order to determine the accurate analysis.

\section{Conclusion}

We did not observe any statistically significant adverse effect proportion in patients undergoing the Fontan procedure according to ventricle dominancy except for prolonged pleural effusion. As a consequence, we state that ventricle dominancy does not impact early postoperative outcomes following the Fontan procedure. 


\section{Ethics}

Ethics Committee Approval: This study was approved by the local ethical committee with decision number $18-10.2 \mathrm{~T} / 43$ on $31 / 10 / 2018$.

Informed Consent: The informed consent form was obtained from each patient.

Peer-review: Internally and externally peer-reviewed.

\section{Authorship Contributions}

Surgical and Medical Practices: Ç.B., G.A., O.N.T., Y.A., Concept: Ç.B., Design: G.A., Data Collection or Processing: G.A., O.N.T., Analysis or Interpretation: Ç.B., Y.A., Literature Search: Ç.B., G.A., O.N.T., Writing: Ç.B., G.A.

Conflict of Interest: No conflict of interest was declared by the authors.

Financial Disclosure: The authors declared that this study received no financial support.

\section{References}

1. Fontan F, Baudet E. Surgical repair of tricuspid atresia. Thorax 1971;26:2408.

2. Galantowicz M, Cheatham JP. Lessons learned from the development of a new hybrid strategy for the management of hypoplastic left heart syndrome. Pediatr Cardiol 2005;26:190-9.

3. d'Udekem Y, Xu MY, Galati JC, et al. Predictors of survival after singleventricle palliation:the impact of right ventricular dominance. J Am Coll Cardiol 2012;59:1178-85.

4. d'Udekem Y, Iyengar AJ, Galati JC, et al. Redefining expectations of longterm survival after the Fontan procedure: twenty-five years of followup from the entire population of Australia and New Zealand. Circulation 2014;130:32-8.
5. Giardini A, Hager A, Pace Napoleone C, Picchio FM. Natural history of exercise capacity after the Fontan operation: a longitudinal study. Ann Thorac Surg 2008;85:818-21.

6. Khairy P, Poirier N, Mercier LA. Univentricular heart. Circulation 2007; 115:800-12. 2.

7. Backer CL. The functionally univentricular heart: which is better-right or left ventricle? J Am Coll Cardiol 2012;59:1186-7.

8. Kamata M, Stiver C, Naguib A, Tumin D, Tobias JD. A Retrospective Analysis of the Influence of Ventricular Morphology on the Perioperative Outcomes After Fontan Surgery. Journal of cardiothoracic and vascular anesthesia, 2017;31:128-33.

9. Alsoufi B, Gillespie S2, Kim D2, et al. The impact of dominant ventricle morphology on palliation outcomes of single ventricle anomalies. Ann Thorac Surg 2016;102:593-601.

10. Hirsch JC, Goldberg C, Bove EL, et al. Fontan operation in the current era. A 15-year single institution experience. Ann Surg 2008;248:402-10.

11. Tweddell JS, Nersesian M, Mussatto KA, et al. Fontan palliation in the modern era: factors impacting mortality and morbidity. Ann Thorac Surg 2009;88:1291-9.

12. Iyengar, AJ, Winlaw DS, Galati JC, et al. The extracardiac conduit Fontan procedure in Australia and New Zealand: hypoplastic left heart syndrome predicts worse early and late outcomes. Eur J Cardiothorac Surg 2014;4:465-73; discussion 473.

13. Lemler MS, Scott WA, Leonard SR, Stromberg D, Ramaciotti C. Fenestration improves clinical outcome of the Fontan procedure: a prospective, randomized study. Circulation 2002;105:207-12.

14. McGuirk SP, Winlaw DS, Langley SM, et al. The impact of ventricular morphology on midterm outcome following completion total cavopulmonary connection. Eur J Cardiothorac Surg 2003;24:37-46.

15. Blinder JJ, Goldstein SL, Lee VV, et al. Congenital heart surgery in infants: effects of acute kidney injury on outcomes. J Thorac Cardiovasc Surg 2012;143:368-74.

16. Taylor M L, Carmona F, Thiagarajan RR, et al. Mild post- operative acute kidney injury and outcomes after surgery for congenital heart disease. J Thorac Cardiovasc Surg 2013;146:146-52. 